\title{
Prescrição de medicamentos potencialmente inapropriados para idosos: um estudo transversal em instituição psiquiátrica
}

\author{
Prescription of potentially inappropriate medications for older \\ adults: a cross-sectional study in psychiatric institution
}

Kaio Vinicius Freitas de Andrade', Cintya da Silva Filho², Letícia Lima Junqueira²

\section{RESUMO}

Objetivo: Estimar a frequência de prescrição de medicamentos potencialmente inapropriados (MPIs) para idosos institucionalizados em um hospital psiquiátrico. Métodos: Estudo descritivo, transversal, baseado em análise de prescrições médicas para indivíduos com 65 anos ou mais ( $n=40$ ), elaboradas no mês de janeiro de 2013 e coletadas de prontuários médicos, em uma instituição hospitalar pública especializada em saúde mental. Foram analisadas variáveis sociodemográficas e clínicas relativas ao idoso. Os medicamentos prescritos foram categorizados, pelos Critérios de Beers (CB), em: 1) MPIs que devem ser evitados em qualquer condição clínica; 2) MPIs nas condições clínicas identificadas no estudo; 3) medicamentos que devem ser utilizados com precaução em idosos. Resultados: Houve predomínio do sexo feminino (62,5\%, $n=25 / 40)$. Em relação à idade, a média foi de 73 anos (66-93 anos). 0 tempo médio de internamento foi de 15 anos, e o principal diagnóstico foi de esquizofrenia $(56,4 \% ; n=22 / 39)$. A prevalência de polifarmácia foi de $70 \%$. Os medicamentos mais prescritos foram prometazina e haloperidol. Em relação aos CB, observou-se que 40,2\% ( $n=90 / 224)$ dos medicamentos prescritos foram classificados como MPIs em qualquer condição clínica; $38,8 \%$ ( $n=87 / 224)$ foram considerados como MPIs em determinadas condições clínicas e

\section{Palavras-chave}

Medicamentos sob

prescrição, saúde mental, hospitais psiquiátricos, prescrição médica.

$23,7 \%$ ( $n=53 / 224)$ pertenciam à categoria dos medicamentos que devem ser utilizados com cautela em idosos. Conclusão: Obteve-se elevada prevalência de MPIs para idosos, quando comparada com estudos semelhantes. Os CBs contribuíram para a análise das prescrições, possibilitando a identificação de medicamentos que podem potencializar os riscos de desenvolvimento de iatrogenias ou agravamento de patologias preexistentes.

\section{ABSTRACT}

Objective: To estimate the frequency of prescription of potentially inappropriate medications (PIM) for institutionalized older adults in a psychiatric hospital. Methods: Descriptive study, transversal, based on analysis of prescriptions for individuals aged over 65 years $(n=40)$, drawn up in January 2013 and collected from medical records in a public hospital. Sociodemographic and clinical variables were analyzed. Medications were categorized by Beers Criteria (BC): 1) PIM which should be avoided in any clinical condition; 2) PIM to avoid in older adults with certain diseases identified on the study; 3 ) medications to be used with caution in older adults.

1 Universidade Estadual de Feira de Santana (UEFS), Departamento de Saúde.

2 Universidade Estadual de Feira de Santana (UEFS).

Recebido em

$4 / 6 / 2015$

Aprovado em

24/5/2016 


\section{Keywords}

Prescription drugs, mental health, psychiatric hospitals, medical prescription.
Results: There was a predominance of females $(62.5 \%, n=25 / 40)$. Regarding age, the average was 73 years (66-93 years). The inpatient average time was 15 years and the main diagnosis was schizophrenia (56.4\%; $n=22 / 39$ ). The prevalence of polypharmacy was $70 \%$. The most commonly prescribed drugs were promethazine and haloperidol. Regarding the $\mathrm{CB}$, it was observed that $40.2 \%$ ( $n=90 / 224$ ) of the prescribed drugs have been classified as PIM in any clinical condition; 38.8\% ( $n=87 / 224)$ PIM in specific clinical conditions and 23.7\% ( $n=53 / 224)$ belonged to the category of drugs that should be used with caution in the older adults. Conclusion: We obtained a high prevalence of PIM for older adults compared with similar studies. The $\mathrm{BC}$ contributed to the analysis of the requirements, enabling the identification of drugs that may enhance the risk of developing iatrogenic or worsening of preexisting conditions.

\section{INTRODUÇÃO}

Medicamentos potencialmente inapropriados (MPIs) são aqueles cujos riscos associados à sua utilização podem ser superiores aos benefícios terapêuticos'. Apesar das evidências associadas com desfechos negativos, eles continuam a ser prescritos e utilizados em idosos².

O uso de MPI é considerado como um dos fatores de risco mais importantes para eventos adversos com medicamentos em idosos ${ }^{3}$. Esse fenômeno também está associado com a complexidade dos problemas de saúde nessa população, que geralmente requerem a prescrição de múltiplos fármacos, conceituada como polifarmácia. Somam-se a esse fato as alterações farmacocinéticas e farmacodinâmicas inerentes ao envelhecimento ${ }^{4,5}$.

A polifarmácia é comum entre idosos e mostra-se associada com o sexo feminino, aumento da idade, hospitalização, consultas com diferentes prescritores, automedicação, baixa percepção de saúde e qualidade de vida, além do acometimento por doenças crônico-degenerativas ${ }^{3-5}$.

Em 1990, nos países desenvolvidos, foram produzidos instrumentos capazes de identificar padrões inadequados de prescrições e problemas farmacoterapêuticos, com base em critérios implícitos, explícitos ou a combinação de ambos ${ }^{5-7}$.

Os critérios implícitos baseiam-se em revisões terapêuticas individualizadas, cuja validade e confiabilidade são de difícil avaliação ${ }^{6}$. Os critérios explícitos foram elaborados por meio de consensos entre profissionais, que estabeleceram índices de adequação para identificar drogas de alto risco e inapropriadas, resultando na elaboração de listas de MPIs, além de sugestões alternativas terapêuticas mais seguras².

Os critérios usualmente empregados para esse tipo de avaliação foram propostos por Beers et al. em 1991 e atualizados em 1997, 2003 e 20122,6-8. Os Critérios de Beers (CB), publicados inicialmente em 1991, possibilitaram a identificação do uso inadequado de medicamentos em idosos institucionalizados nos Estados Unidos. Consistiam em uma lista contendo 19 medicamentos ou grupos farmacológicos que deveriam ser evitados e 11 medicamentos cuja dose, frequência de uso ou duração do tratamento não deveriam ser excedidas. A lista foi obtida por meio de consenso entre peritos em medicina geriátrica e farmacologia, utilizando-se o método Delphi modificado 6 .

Em 1997, os CB foram ampliados para aplicação em idosos não institucionalizados e incorporaram tópicos adicionais sobre o uso de medicamentos em idosos com patologias específicas, resultando em uma lista com 28 medicamentos ou classes de medicamentos inadequados e 35 medicamentos ou classes de medicamentos inadequados em 15 condições patológicas específicas?.

Em 2003, Fick et al. atualizaram os CB, alterando sua denominação para Critérios de Beers-Fick. Os medicamentos foram então categorizados em: 1) medicamentos ou grupos farmacológicos que devem ser evitados em idosos independentemente do diagnóstico ou condição clínica; 2) medicamentos ou grupos farmacológicos que não devem ser empregados em determinadas circunstâncias clínicas. Definiu-se uma lista com 48 medicamentos inadequados e outra contendo medicamentos inapropriados em indivíduos com 20 condições patológicas específicas ${ }^{8}$.

Em 2012, os critérios passaram por nova atualização, seguindo uma abordagem baseada em evidências. A nomenclatura utilizada passou novamente a ser Critérios de Beers, contendo 53 medicamentos ou classes de medicamentos, agrupados em três categorias: 1) MPIs que devem ser evitados em idosos; 2) MPIs para idosos com determinadas condições clínicas; 3) medicamentos que devem ser utilizados com precaução em idosos².

Desde então, diversos estudos vêm sendo desenvolvidos com base nesses critérios, considerando que a frequência de prescrição de MPIs para idosos pode atuar como um indicador de qualidade assistencial em instituições hospitalares e/ou ambulatoriais, com relação à segurança da farmacoterapia ${ }^{9}$. Nesse contexto, objetivou-se estimar a frequência de prescrição de MPIs para idosos institucionalizados no Hospital Especializado Lopes Rodrigues (HELR), em Feira de Santana, Bahia, Brasil.

\section{MÉTODOS}

Estudo descritivo, transversal, cuja unidade de análise foram prescrições de medicamentos para idosos com 65 anos ou 
mais, no mês de janeiro 2013, em processo de internação no HELR, uma instituição hospitalar pública especializada em saúde mental, com 273 leitos de internação, localizada em Feira de Santana, Bahia, município com 556.642 habitantes ${ }^{10}$.

Os critérios para inclusão da prescrição no estudo foram: 1) idade $\geq 65$ anos; 2) ter sido elaborada em janeiro de 2013; 3) estar escrita de forma legível, permitindo a leitura e decodificação completa. Foram descritas variáveis sociodemográficas (sexo e idade) e clínicas (diagnóstico que motivou a internação; comorbidades; tempo de internação; medicamentos prescritos).

Elegeu-se como cenário de estudo a unidade geriátrica da referida instituição, que contava com 78 leitos de internação no período da coleta. Com base nos critérios de inclusão, foram selecionados 40 prontuários, sendo analisada 1 (uma) prescrição por prontuário. Quando havia mais de uma prescrição elaborada no mês de estudo, incluiu-se apenas aquela com maior número de medicamentos.

Os medicamentos prescritos foram classificados segundo os $\mathrm{CB}^{2}$ em: 1) MPls que devem ser evitados em qualquer condição clínica; 2) MPIs nas condições clínicas identificadas no estudo; 3 ) medicamentos que devem ser utilizados com precaução em idosos.

As informações foram coletadas pelo pesquisador principal e, posteriormente, tabuladas e analisadas por todos os pesquisadores. Realizou-se uma análise descritiva por meio do software SPSS para Windows 18.011.

As recomendações da Resolução no 466/2012, do Conselho Nacional de Saúde ${ }^{12}$, foram atendidas. A pesquisa foi autorizada pelo Comitê de Ética em Pesquisa da Universidade Estadual de Feira de Santana (CEP/UEFS), Bahia, com Parecer no 186.574 (CAAE: 08981312.9.0000.0053).

\section{RESULTADOS}

Observou-se maior frequência de indivíduos do sexo feminino $(62,5 \% ; n=25 / 40)$. A idade média foi de 73 anos, com desvio-padrão de 6,5 (variando de 66-93 anos). O tempo médio de internamento foi de 15 anos, com desvio-padrão de 4,3 (2-18 anos). Esquizofrenia (56,4\%; $n=22 / 39)$ foi o diagnóstico mais prevalente. As principais comorbidades foram hipertensão arterial $(32,6 \% ; n=16 / 49)$ e dislipidemia $(12,3 \%$; $\mathrm{n}=6 / 49)$ (Tabela 1).

No total, foram prescritos 224 medicamentos (com 57 princípios ativos distintos), obtendo-se uma média de 5,6 medicamentos/prescrição (1-9 medicamentos). A prevalência de polifarmácia (cinco ou mais medicamentos/prescrição) foi de 70\% ( $n=28 / 40)$. Dos 57 fármacos, 33,3\% ( $n=$ 19/57) eram inapropriados em qualquer condição clínica, com nível elevado de evidência e forte grau de recomendação contrária ao uso em idosos.
Tabela 1. Caracterização dos idosos de acordo com o sexo, diagnóstico principal e comorbidades - Feira de Santana, Bahia, janeiro de 2013

\begin{tabular}{lcc}
\hline & $\mathbf{n}$ & $\%$ \\
\hline Sexo ( $\mathbf{n}=40)$ & 25 & 62,5 \\
Feminino & 15 & 37,5 \\
Masculino & & \\
Diagnóstico principal ( $\mathrm{n}=39)$ & 22 & 56,4 \\
Esquizofrenia (F20) & 11 & 28,1 \\
Psicose orgânica não especificada (F29) & 3 & 7,7 \\
Retardo mental moderado (F71) & 1 & 2,6 \\
Retardo mental grave (F72) & 1 & 2,6 \\
Transtornos esquizoafetivos (F25) & 1 & 2,6 \\
Tricotilomania (F63.3) & & \\
Comorbidades (n=49) & 16 & 32,6 \\
Hipertensão arterial & 6 & 12,3 \\
Dislipidemia & 5 & 10,2 \\
Diabetes mellitus tipo II & 22 & 44,9 \\
Outras & & \\
\hline
\end{tabular}

Os medicamentos mais prescritos foram prometazina $(11,6 \% ; n=26 / 224)$ e haloperidol $(10,3 \% ; n=23 / 224)$. Desses, $40,2 \%$ ( $n=90 / 224)$ eram inapropriados em qualquer condição clínica; $38,8 \%$ ( $n=87 / 224)$ ), nas condições clínicas dos indivíduos que receberam a prescrição e 23,7\% ( $n=53 / 224)$ deveriam ser utilizados com cautela em idosos (Tabela 2).

O número médio de $\mathrm{MPI}$ em qualquer condição clínica por prescrição $(n=90 / 40)$ foi 2,25 (variando de 1-5). Em 90\% ( $n=36 / 40$ ) das prescrições havia pelo menos 1 (um) MPI desse tipo. Nas prescrições em que houve polifarmácia $(n=28)$, em $96,4 \%$ ( $n=27 / 28$ ) havia pelo menos 1 (um) MPI em qualquer condição clínica e em 92,8\% ( $n=26 / 28)$ havia pelo menos 1 (um) MPI na condição clínica específica dos indivíduos.

Tabela 2. Frequência de medicamentos prescritos segundo Critérios de Beers ( $n=224$ ) - Feira de Santana, Bahia, janeiro de 2013

\begin{tabular}{lcc}
\hline & $\mathbf{n}$ & $\%$ \\
\hline MPI em qualquer condição clínica & 134 & 59,8 \\
Não & 90 & 40,2 \\
Sim & & \\
MPI em determinadas condições & 137 & 61,2 \\
Não & 87 & 38,8 \\
Sim & & \\
Uso com precaução* & 171 & 76,3 \\
Não & 53 & 23,7 \\
Sim &
\end{tabular}

* Medicamentos que devem ser utilizados com cautela em idosos, quando os benefícios superarem os riscos, e que requerem monitoramento de eventos adversos, segundo a American Geriatrics Society, 2012. 
A prescrição de MPI em qualquer condição clínica foi mais prevalente na faixa etária de 65-79 anos $(70,0 \% ; n=28 / 40) \mathrm{e}$ no sexo feminino $(57,5 \% ; n=23 / 40)$. Dentre esses medicamentos ( $n=90)$, destacaram-se os antipsicóticos (36,7\%; $n=$ 33/90), anti-histamínicos de uso sistêmico (28,9\%; $n=26 / 90)$ e ansiolíticos (13,3\%; $n=12 / 90)$. O MPIs mais prescritos foram: prometazina $(26,7 \% n=24 / 90)$, haloperidol $(25,5 \% ; n=$ 23/90) e diazepam (12,2\%; $n=11 / 90)$ (Tabela 3).

Dos 57 fármacos distintos entre os medicamentos prescritos ( $n=224), 31,6 \%(n=18 / 57)$ foram classificados como inapropriados em determinadas doenças ou síndromes. Os mais prescritos foram prometazina $(27,6 \% ; n=24 / 87)$, haloperidol $(26,4 \% ; n=23 / 87)$ e clonazepam $(8,0 \% ; n=7 / 57)$.

Em 80\% ( $n=32 / 40)$ das prescrições analisadas constava pelo menos 1 (um) medicamento que deve ser usado com precaução em idosos, prevalecendo antipsicóticos (62,3\%; n $=33 / 53)$, anticonvulsivantes $(20,7 \% ; n=11 / 53)$, antidepressi$\operatorname{vos}(15,1 \% ; n=8 / 53)$ e antitrombóticos $(1,9 \% ; n=1 / 53)$. Entre os medicamentos prescritos nesse grupo, prevaleceram o haloperidol $(26,4 \% ; n=14 / 53)$ e a carbamazepina $(20,7 \%$; $n=11 / 53)$.

Tabela 3. Caracterização dos MPI em qualquer condição clínica $(n=90)$ - Feira de Santana, Bahia, janeiro de 2013

\begin{tabular}{lcc}
\hline & $\mathbf{n}$ & $\%$ \\
\hline Grupo farmacológico & 33 & 36,7 \\
Antipsicóticos & 26 & 28,9 \\
Anti-histamínicos de uso sistêmico & 12 & 13,3 \\
Ansiolíticos & 19 & 21,1 \\
Outros & & \\
Medicamentos prescritos & 24 & 26,7 \\
Prometazina & 23 & 25,5 \\
Haloperidol & 11 & 12,2 \\
Diazepam & 7 & 7,8 \\
Clonazepam & 6 & 6,7 \\
Clorpromazina & 19 & 21,1 \\
$\quad$ Outros & & \\
\hline
\end{tabular}

\section{DISCUSSÃO}

A polifarmácia entre idosos associa-se com a elevada carga de doenças crônicas e manifestações clínicas resultantes do envelhecimento ${ }^{13,14}$. Seus desfechos podem contribuir para a redução da segurança da terapia medicamentosa, com prevalência variando de $59,5 \%$ a $71,8 \%{ }^{15,16}$.

No presente estudo, a prescrição de MPI em qualquer condição clínica foi elevada, em comparação com prevalências de $41 \%$ e $54,1 \%$, obtidas em dois estudos semelhantes ${ }^{15,17}$. Essa diferença também foi observada na comparação com estudos internacionais, que obtiveram frequências de pelo menos um MPI em quaisquer condições clínicas, iguais a $38 \%$ e $46 \%{ }^{18}$.

Em análise de prescrições médicas realizada em Portugal ( $n=291)$, evidenciou-se, entre os MPIs mais prescritos, predomínio de antipsicóticos $(27,5 \%)$ e benzodiazepínicos $(25,1 \%)$, padrão semelhante ao encontrado no presente estudo. Relativamente aos princípios ativos considerados inapropriados, o haloperidol esteve entre os mais prescritos ${ }^{19}$.

Os antipsicóticos aparecem nas três categorias de $\mathrm{MPIs}^{2}$. Esses fármacos são lipossolúveis, podendo acumular-se progressivamente no idoso, em decorrência da elevação proporcional da gordura corporal associada ao envelhecimento. Outros fatores como redução dos níveis de albumina plasmática e das taxas de metabolismo hepático e excreção renal contribuem para o aumento dos tempos de meia-vida e maior risco de toxicidade ${ }^{20}$. No presente estudo, a maioria dos sujeitos apresentou esquizofrenia. Nessa população, pela própria natureza do transtorno, a prevalência de prescrição de antipsicóticos costuma ser mais elevada, podendo ter como consequência maior frequência de eventos adversos.

Sintomas extrapiramidais, hipotensão ortostática e efeitos anticolinérgicos decorrentes do uso de antipsicóticos podem comprometer a mobilidade dos idosos ${ }^{21,22}$. Os antipsicóticos convencionais podem aumentar o risco de acidente vascular cerebral e a mortalidade em idosos com demência ${ }^{23}$. Também podem ocasionar prejuízos na função psicomotora, levando à ataxia, que favorece a ocorrência de quedas e fraturas².

Recomenda-se evitar a prescrição de antipsicóticos para idosos, reservando-os para quadros com sintomatologia mais grave e que não responderam adequadamente a outras alternativas, empregando-se as menores doses necessárias para a obtenção dos efeitos terapêuticos. O risco de eventos adversos graves, principalmente cerebrovasculares, deve ser avaliado ${ }^{24}$.

Prometazina e difenidramina são anti-histamínicos utilizados como sedativos e hipnóticos, isoladamente ou em associação com outros fármacos. Possuem propriedades anticolinérgicas intensas, provocando confusão e disfunção cognitiva no idoso. A prometazina possui efeito sedativo prolongado e tolerância reduzida com o avanço da idade, estando associada à ocorrência de confusão, boca seca, constipação e retenção urinária em idosos. É, ainda, inapropriada em determinadas condições clínicas, como na doença de Parkinson, por agravar os sintomas parkinsonianos².

Na maioria dos hospitais psiquiátricos brasileiros, a prometazina foi consagrada como um dos fármacos mais prescritos no tratamento das manifestações extrapiramidais distônicas agudas ocasionadas pelos antipsicóticos típicos ${ }^{25}$. No entanto, deve-se atentar para o risco de deflagração de estados confusionais e o agravamento de possíveis déficits cognitivos; nos casos de efeitos extrapiramidais relacionados ao uso de antipsicóticos em idosos, recomenda-se a redução 
da dose ou a substituição do medicamento por outros que não ocasionam efeitos anticolinérgicos como loratadina, desloratadina, cetirizina e azelastina ${ }^{26}$.

Em razão da menor incidência de efeitos adversos extrapiramidais em relação aos antipsicóticos tradicionais ou típicos, os antipsicóticos atípicos, eficazes no controle das manifestações psicóticas em adultos e idosos, passaram a ser utilizados com maior frequência e como primeira opção terapêutica para o manejo dos sintomas psicóticos e comportamentais, tornando dispensável a associação com anti-histamínicos ou anticolinérgicos ${ }^{24}$.

O diazepam, o clordiazepóxido e o clonazepam são benzodiazepínicos de longa ação classificados como MPIs em qualquer condição clínica e a depender da doença ou síndrome. Possuem meia-vida longa em idosos, produzindo sedação prolongada, alterações psicomotoras, falta de coordenação, aumento do risco de quedas e fraturas, além de delírios, perturbações cognitivas e elevação da mortalidade ${ }^{2,27,28}$.

Entre os benzodiazepínicos, em 2003, faziam parte da lista de MPIs apenas os de curta ação, caso fossem prescritas doses elevadas, e os de longa duração, uma vez que os primeiros não promovem a geração de metabólitos ativos e, por isso, foram associados a menor risco para os idosos. Porém, na atualização mais recente, em 2012, todos os benzodiazepínicos foram incluídos na categoria de MPIs, independentemente da sua duração de ação' ${ }^{19}$.

Embora os benzodiazepínicos sejam amplamente utilizados em idosos, não se recomenda o seu uso, pelo fato de que, com o avanço da idade, há alterações qualitativas e quantitativas no complexo gabaérgico, particularmente em relação aos receptores GABA, que são responsáveis pelo aumento da sensibilidade aos benzodiazepínicos ${ }^{29}$. São necessárias doses mais baixas e níveis plasmáticos inferiores nos idosos do que entre os jovens, para alcançar o mesmo nível de sedação. A prescrição de benzodiazepínicos para idosos só deve ocorrer quando for extremamente necessária, dando-se sempre preferência aos fármacos de meia-vida curta e intermediária. Existem fármacos que podem ser prescritos como alternativa aos benzodiazepínicos em casos de ansiedade, como a buspirona, que possui efeito ansiolítico sem provocar sedação, dependência e síndrome de abstinência.

Entre os antiepilépticos, o fenobarbital é classificado como MPI para idosos em qualquer condição clínica, por causa da alta taxa de dependência física e risco de overdose, mesmo em baixas dosagens. Os barbitúricos são fortemente aditivos e causam mais efeitos adversos quando utilizados como anticonvulsivantes nos idosos do que a maioria dos sedativos ou hipnóticos. A população idosa requer dosagens menores para atingir as mesmas concentrações séricas de fenobarbital que os adultos ${ }^{30}$. Recomenda-se evitar a prescrição de fenobarbital para idosos, preferindo-se a gabapentina como agente de primeira escolha, já que é provavelmente o fármaco mais bem tolerado por essa população 31,32 .

\section{CONCLUSÕES}

Apesar de a prescrição de MPIs também ser um problema frequente nas unidades especializadas em psiquiatria, poucos estudos investigaram com profundidade o valor terapêutico e os riscos associados ao uso de medicamentos em idosos assistidos nessas instituições.

O principal achado do presente estudo consiste na elevada prevalência de MPI em prescrições para idosos, quando comparada a outros estudos com metodologia semelhante. Conclui-se que os Critérios de Beers contribuíram para a análise das prescrições selecionadas, possibilitando a identificação de medicamentos que podem potencializar os riscos de desenvolvimento de iatrogenias ou agravamento de patologias preexistentes.

Embora os Critérios de Beers sejam utilizados com frequência em pesquisas nacionais e internacionais, uma das limitações apresentadas na execução do estudo foi a escassez de estudos cuja análise tenha sido pautada na atualização mais recente dos referidos critérios. Outras limitações de ordem metodológica podem ter contribuído para reduzir a validade externa do estudo, em especial o número relativamente pequeno de prescrições analisadas e a natureza do estudo.

\section{CONTRIBUIÇÕES INDIVIDUAIS}

Kaio Vinicius Freitas de Andrade - Elaboração do projeto de pesquisa e desenho do estudo, análise de dados, interpretação dos resultados, elaboração de tabelas, redação e revisão final do artigo.

Cintya da Silva Filho - Redação e formatação do artigo e das tabelas, revisão do conteúdo.

Leticia Lima Junqueira - Coleta, processamento, análise dos dados e elaboração do artigo.

\section{CONFLITOS DE INTERESSE}

Os autores não possuem conflitos de interesse a serem declarados.

\section{REFERÊNCIAS}

1. Hanlon JT, Schmader KE, Samsa GP, Weinberger M, Uttech KM, Lewis IK, et al. A method for assessing drug therapy appropriateness. J Clin Epidemiol. 1992;45(10):1045-51.

2. American Geriatrics Society 2012 Beers Criteria Update Expert Panel. American Geriatrics Society updated Beers Criteria for potentially inappropriate medication use in older adults. J Am Geriatr Soc. 2012;60(4):616-31.

3. Ribeiro AQ, Araújo CMC, Acurcio FA, Magalhães SMS, Chaimowicz, F. Qualidade do uso de medicamentos por idosos: uma revisão dos métodos de avaliação disponíveis. Ciênc Saúde Coletiva. 2005;10(4):1037-45 
4. Fernández BN, Diaz CM, Polo VDR, Martín AG, Ruiz BC. Global therapeutic perspective in geriatric patients. Electron J Biomed. 2006;3:29-38.

5. Gallagher PF, Barry PJ, Ryan C, Hartigan I, O'Mahony D. Inappropriate prescribing in an acutely ill population of elderly patients as determined by Beers' Criteria. Age Ageing. 2008;37(1):96-101.

6. Beers MH, Ouslander JG, Rollingher I, Reuben DB, Brooks J, Beck JC. Explicit criteria for determining inappropriate medication use in nursing home residents. UCLA Division of Geriatric Medicine. Arch Intern Med. 1991;151(9):1825-32

7. Beers MH. Explicit criteria for determining potentially inappropriate medication use by the elderly. An update. Arch Intern Med. 1997;157(14):1531-6.

8. Fick D, Semla T. Improving medication use in gerontological nursing: now is the time for interdisciplinary collaboration and translation. J Gerontol Nurs. 2011;37(8):3-4.

9. Garfinkel D, Mangin D. Feasibility study of a systematic approach for discontinuation of multiple medications in older adults: addressing polypharmacy. Arch Intern Med. 2010;170(18):1648-54.

10. Instituto Brasileiro de Geografia e Estatística (IBGE). Distribuição da população por sexo, segundo os grupos de idade, Feira de Santana (BA). Censo 2010. Disponível em: < http:// www.cens02010.ibge.gov.br/sinopse/webservice/frm_piramide.php?codigo $=291080 \& \mathrm{c}$ orhomem $=3 \mathrm{~d} 4590 \&$ cormulher $=9 \mathrm{cdbfc}>$. Acesso em: 27 maio 2013.

11. Statistical Package for the Social Sciences Inc. (SPSS). Released 2009. PASW Statistics for Windows, Version 18.0. Chicago: SPSS Inc.; 2009.

12. Brasil. Conselho Nacional de Saúde. Resolução n 466, de 12 de dezembro de 2012. Aprova as diretrizes e normas regulamentadoras de pesquisas envolvendo seres humanos. Diário Oficial da União 2013.

13. Secoli SR. Polifarmácia: interações e reações adversas no uso de medicamentos por idosos. Rev Bras Enfermagem 2010;63(1):136-40.

14. Linjakumpu T, Hartikainen S, Klaukka T, Veijola J, Kivelä SL, Isoaho R. Use of medications and polypharmacy are increasing among the elderly. J Clin Epidemiol. 2002;55(8): 809-17.

15. Sousa-Muñoz RL, Ibiapina GR, Gadelha CS, Maroja JLS. Prescrições geriátricas inapropriadas e polifarmacoterapia em enfermarias de clínica médica de um Hospital-Escola. Rev Bras Geriatr Gerontol. 2012;15(2):315-23.

16. Padilha KLRM, Pereira AGL, Escosteguy CC, Matos HJ, Ferreira CSMN, Chaves SMC. Polifarmácia no idoso: um estudo piloto em pacientes do Serviço de Geriatria do HSE. Boletim Epidemiológico nº 38 - Hospital Federal dos Servidores do Estado; 2009.
17. Gorzoni ML, Fabbri RMA, Pires SL. Medicamentos em uso à primeira consulta geriátrica. Diagn Tratamento. 2006;11(3):138-42.

18. Soares MAS. Avaliação da terapêutica potencialmente inapropriada no doente geriátrico [tese]. Lisboa: Universidade de Lisboa, Faculdade de Farmácia; 2009.

19. Vaz CSSB. Medicamentos potencialmente inapropriados em idosos: a realidade de um serviço de medicina [dissertação]. Coimbra: Faculdade de Farmácia, Universidade de Coimbra; 2012.

20. Bowie MW, Slattum PW. Pharmacodynamics in older adults: a review. Am J Geriatr Pharmacother. 2007;5(3):263-303.

21. Timiras PS. Physiological basis of aging and geriatrics. 4th ed. Boca Raton: CRC Press; 2007.

22. Koda-Kimble M, Young LY, Kradjan WA, Guglielmo BJ, Alldredge, BK, Corelli RL. Applied therapeutics: the clinical use of drugs. 8th ed. New York: Lipincott Williams \& Wilkins; 2005.

23. Pham CB, Dickman RL. Minimizing adverse drug events in older patients. Am Fam Physician. 2007;76(12):1837-44

24. Forlenza OV, Cretaz E, Diniz BSO. 0 uso de antipsicóticos em pacientes com diagnóstico de demência. Rev Bras Psiquiatr. 2008;30(3):265-70.

25. Soares MA, Fernandez-Llimós F, Lança C, Cabrita J, Morais JA. Operacionalização para Portugal: Critérios de Beers de medicamentos inapropriados nos doentes idosos. Acta Med Port. 2008;21(5):441-52.

26. Frota LH. Cinquenta anos de medicamentos antipsicóticos em psiquiatria. J Bras Psiquiatr. 2001;50(5/6):213-30

27. Wells BG, Dipiro JT, Schwinghammer TL, Dipiro CV. Pharmacotherapy handbook. 7th ed. New York: McGraw-Hill; 2009.

28. Vicens C, Socias I, Mateu C, Leiva A, Bejarano F, Sempere E, et al. Comparative efficacy of two primary care interventions to assist withdrawal from long term benzodiazepine use: a protocol for a clustered, randomized clinical trial. BMC Fam Pract. 2011;12:23.

29. Turnheim K. Drug therapy in the elderly. Exp Gerontol. 2004;39(11-12):1731-8.

30. Silvado C. Farmacogenética e antiepilépticos (farmacologia das drogas antiepilépticas: da teoria à prática). J Epilepsy Clin Neurophysiol. 2008;14(Supl 2):51-66.

31. Glauser T, Ben-Menachem E, Bourgeois B, Cnaan A, Chadwick D, Guerreiro C, et al. ILAE treatment guidelines: evidence-based analysis of antiepileptic drug efficacy and effectiveness as initial monotherapy for epileptic seizures and syndromes. Epilepsia. 2006:47(7):1094-120

32. Sthepen LJ, Brodie MJ. Epilepsy in elderly people. Lancet. 2000;355(9213):1441-6. 